\title{
A computational fluid dynamics investigation of the segmented integral squeeze film damper
}

\author{
Petr Ferfecki ${ }^{1,2, *}$, Jaroslav Zapoměl ${ }^{2,3}$, Marek Gebauer ${ }^{1}$, Václav Polreich ${ }^{4}$, and Jiři Křenek ${ }^{4}$ \\ ${ }^{1}$ VŠB-Technical University of Ostrava, IT4Innovations National Supercomputing Center, 70833 \\ Ostrava-Poruba, Czech Republic \\ ${ }^{2}$ VŠB-Technical University of Ostrava, Department of Applied Mechanics, 70833 Ostrava-Poruba, \\ Czech Republic \\ ${ }^{3}$ Institute of Thermomechanics of the CAS, v. v. i., Department of Dynamics and Vibration, 18200 \\ Prague, Czech Republic \\ ${ }^{4}$ Doosan Škoda Power s.r.o., Department of Measurement \& Diagnostics, 30128 Pilsen, \\ Czech Republic
}

\begin{abstract}
Rotor vibration attenuation is achieved with damping devices which work on different, often mutually coupled, physical principles. Squeeze film dampers are damping devices that have been widely used in rotordynamic applications. A new concept of a 5-segmented integral squeeze film damper, in which a flexure pivot tilting pad journal bearing is integrated, was investigated. The damper is studied for the eccentric position between the outer and inner ring of the squeeze film land. The ANSYS CFX software was used for solving the pressure and velocity distribution. The development of the complex three-dimensional computational fluid dynamics model of the squeeze film damper, learning more about the effect of the forces in the damper, and the knowledge about the behaviour of the flow are the principal contributions of this article.
\end{abstract}

\section{Introduction}

Unbalance of rotating machines is the main source of the lateral vibration of rotors that can be significantly reduced if damping elements are inserted between the rotor and the stationary part. It has been proven both theoretically and experimentally that the damping devices used for vibration suppression can work according to the different, often mutually coupled, physical principles.

The concept of active vibration control for the cantilever beam that is damped by the force generated with the feedback PD controller for the vibration reduction and suppression of the journal bearing instability by the piezo-actuators was reported in [1], respectively [2].

The working principle of the passive or semi-active electromechanical dampers [3] is based on the eddy currents $[4,5]$ generated with two different strategies: (i) changing the reluctance of a magnetic circuit or (ii) moving a conductor in a stationary magnetic field.

${ }^{*}$ Corresponding author: petr.ferfecki@,vsb.cz

Reviewers: Tomáš Lack, Robert Zalewski 
On the other hand, the traditional squeeze film dampers (SFDs) [6, 7] are based on the passive concept of the damping force generation by means of the mutually coupled hydraulic and mechanical physical principles. Main research findings, in the last four decades, on the SFDs and its applications can be found in articles of L. D. Pietra and Adiletta $[8,9]$.

However, in subsequent years the controllable SFDs working in active $[10,11]$ or semiactive concepts [12-19] have been developed. The concept of semi-active working mode accomplished by the electromagnetic principle is represented with SFDs lubricated by electrorheological $[12,13]$ or magnetorheological [16-19] fluids.

The relatively long axial length, the complicated assembling of the squirrel cage spring, and the difficult adjustment of the damper due to the elimination of the deflection of the rotor weight are the main drawbacks of the traditional SFDs. The utilization of the manufacturing process of wire electrical discharge machining has enabled the design of a new segmented integral squeeze film damper (ISFD) [20-24].

In this paper, the complex three-dimensional computational fluid dynamic (CFD) model of the segmented ISFD has been developed. A new concept of a segmented ISFD consisting of 5 segments is studied for the eccentric position between the outer and inner damper ring. The ANSYS CFX software was used for the setup and solution of the CFD model. The pressure and velocity distribution in the segmented ISFD are computed with the mathematical model of the damper, based on the Navier-Stokes equations. The carried-out computational simulations show that the developed CFD model of the segmented ISFD allowed computation of the flow characteristics and the hydraulic forces.

\section{Configurations of the traditional SFDs and the segmented ISFDs}

A traditional SFD basically combines a bearing (generally a rolling-element bearing with elements such as balls or rollers but also different types of bearings such as a tilting pad journal bearing are used) and a thin damping oil film. Figure 1 shows a traditional SFD consisting of a shaft journal, a ball bearing, and damper rings and housing. The shaft journal is supported by a ball bearing, while an anti-rotation of the inner damper ring is provided by the squirrel cage spring that offers a centring elastic mechanism. The outer ring of the bearing is firmly coupled with the damper housing. The thin layer of the oil between the inner and outer ring is fed with oil at a given pressure. The attenuation of the motion is caused by hydrodynamic forces exerted by the squeeze film pressure that is initiated with the precession motions of the shaft journal.

In traditional SFDs, damping is generated by squeezing oil in the damper film and is governed by circumferential film flow as shown in Fig. 1a, which makes it difficult for the control oil flow resistance.

The segmented ISFD (Fig. 1b) design allows partition of the thin layer of the oil film between the inner and outer damper ring into the circumferentially partitioned segments. Partitioning of the squeeze film offers the advantage of a precise damping as well as the stiffness controlled by flow resistance at the end seal clearances as well as the oil supply nozzle $[23,24]$. Therefore, the ISFD can produce damping by the squeezing thin oil film and by the piston/dashpot effect.

The ISFD technology is often integrated into the serial combination of the different types of bearings such as a rolling-element bearing, a sleeve bearing, a flexure pivot tilting pad journal bearing, and other. Benefits from utilizing ISFDs include a simple assembly design of damper, a shorter axial length, a shaft centring ability, and lightweight. 

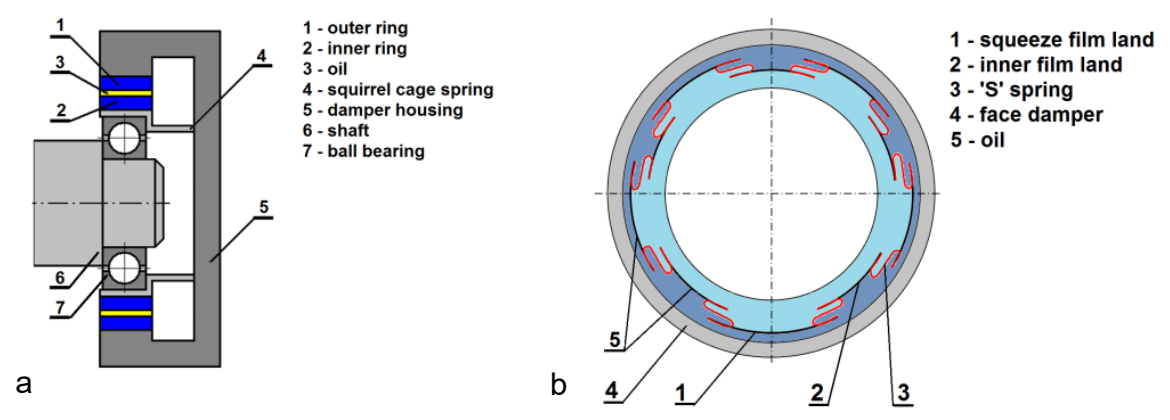

Fig. 1. The scheme of the traditional SFD (a) and the segmented ISFD without a bearing (b)

\section{Governing equations of the flow and the hydraulic forces}

The governing equations for the pressure and velocity distribution in the damper include Navier-Stokes equations and the continuity equation [25], which are set up with the following assumptions: (i) the lubricant behaves as the Newtonian liquid; (ii) the flow in the fluid film is incompressible, laminar, and isothermal

$$
\begin{gathered}
\frac{\partial u}{\partial t}+u \frac{\partial u}{\partial x}+v \frac{\partial u}{\partial y}+w \frac{\partial u}{\partial z}=f_{\mathrm{x}}-\frac{1}{\rho} \frac{\partial p}{\partial x}+\frac{\eta}{\rho}\left(\frac{\partial^{2} u}{\partial x^{2}}+\frac{\partial^{2} u}{\partial y^{2}}+\frac{\partial^{2} u}{\partial z^{2}}\right) \\
\frac{\partial v}{\partial t}+u \frac{\partial v}{\partial x}+v \frac{\partial v}{\partial y}+w \frac{\partial v}{\partial z}=f_{\mathrm{y}}-\frac{1}{\rho} \frac{\partial p}{\partial y}+\frac{\eta}{\rho}\left(\frac{\partial^{2} v}{\partial x^{2}}+\frac{\partial^{2} v}{\partial y^{2}}+\frac{\partial^{2} v}{\partial z^{2}}\right) \\
\frac{\partial w}{\partial t}+u \frac{\partial w}{\partial x}+v \frac{\partial w}{\partial y}+w \frac{\partial w}{\partial z}=f_{\mathrm{z}}-\frac{1}{\rho} \frac{\partial p}{\partial z}+\frac{\eta}{\rho}\left(\frac{\partial^{2} w}{\partial x^{2}}+\frac{\partial^{2} w}{\partial y^{2}}+\frac{\partial^{2} w}{\partial z^{2}}\right) .
\end{gathered}
$$

Where $x, y, z$ are the Cartesian coordinates, which define the directions of the fixed frame of reference, $u, v, w$ are the $x, y, z$ components of the velocity, $f_{\mathrm{x}}, f_{\mathrm{y}}, f_{\mathrm{z}}$ are the components of the body force, $p$ is the pressure, $t$ is the time, and $\rho$ and $\eta$ is the density and dynamic viscosity of the lubricant, respectively.

The equation of continuity represents the conservation of mass, and in the case of the incompressible fluid it can be written in the following form

$$
\frac{\partial u}{\partial x}+\frac{\partial v}{\partial y}+\frac{\partial w}{\partial z}=0
$$

The hydraulic damping force is produced due to squeezing the oil film and by the piston/dashpot effect, and it is obtained with the assumption that the shear stress can be neglected. Therefore, the components of hydraulic force are computed by integration of the pressure distribution around the squeeze and inner film land of all segments and along the length of the damper

$$
f_{\mathrm{y}}=2 R \int_{0}^{\frac{L}{2}} \int_{\varphi_{1}}^{\varphi_{2}} p_{\mathrm{d}} \cos (\varphi) \mathrm{d} \varphi \mathrm{d} Z, \quad f_{\mathrm{z}}=2 R \int_{0}^{\frac{L}{2}} \int_{\varphi_{1}}^{\varphi_{2}} p_{\mathrm{d}} \sin (\varphi) \mathrm{d} \varphi \mathrm{d} Z
$$

$f_{\mathrm{y}}, f_{\mathrm{z}}$ are the $y$ and $z$ components of the damping force, $R$ is the inner ring radius, $p_{\mathrm{d}}$ is the pressure distribution, and $\varphi_{1}, \varphi_{2}$ are the circumferential integration limits.

When the pressure drops down to the atmospheric pressure the cavitation takes place. In accordance with the results of measurements and observations, it is assumed that the pressure of the medium in cavitated areas remains constant, and its magnitude is equal to the pressure in the ambient space [26]. 


\section{Description of the CFD model}

This study deals with the segmented ISFD, which is integrated into the serial combination of the flexure pivot tilting pad journal bearing and was developed in Doosan Group. The damper photography without the flexure pivot tilting pad journal bearing is drawn in Fig. 2.
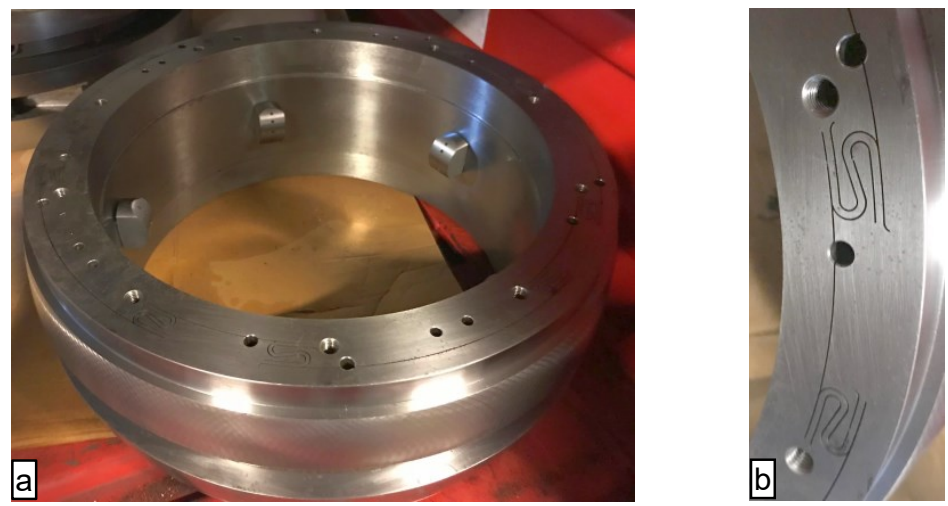

Fig. 2. The image of the body segmented ISFD (a) and the detail of the inner film land with 'S' springs connected the outer and inner damper ring (b)

The CFD model consists of the segmented ISFD without the tilting pad journal bearing. The investigated damper (Fig. 3) has 5 segments (the squeeze film lands), each of them is fed by an oil supply nozzle, and the outflow is controlled by the end seal clearance. The position between the outer and inner damper ring of the squeeze film land (Fig. 4) is offset in the positive z-direction by $0.05 \mathrm{~mm}$, see Fig. 3 .

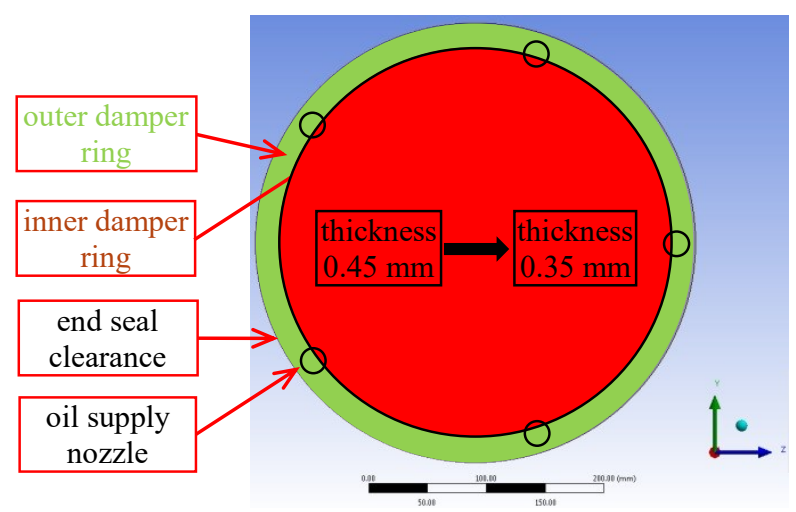

Fig. 3. The configuration of the outer and inner damper ring of ISFD

The technological parameters of the studied damper are as follows: the thickness of the squeeze film land and the inner film land (Fig. 4) is $0.4 \mathrm{~mm}$ in the concentric position, the radius of the outer ring of the squeeze film land is $166 \mathrm{~mm}$, the radius of the outer ring of the inner film land is $153.9 \mathrm{~mm}$, and the length of the damper is $134.5 \mathrm{~mm}$. The lubrication oil density is $870 \mathrm{~kg} \cdot \mathrm{m}^{-3}$ and its dynamic viscosity is $0.0312 \mathrm{~Pa} \cdot \mathrm{s}$. 


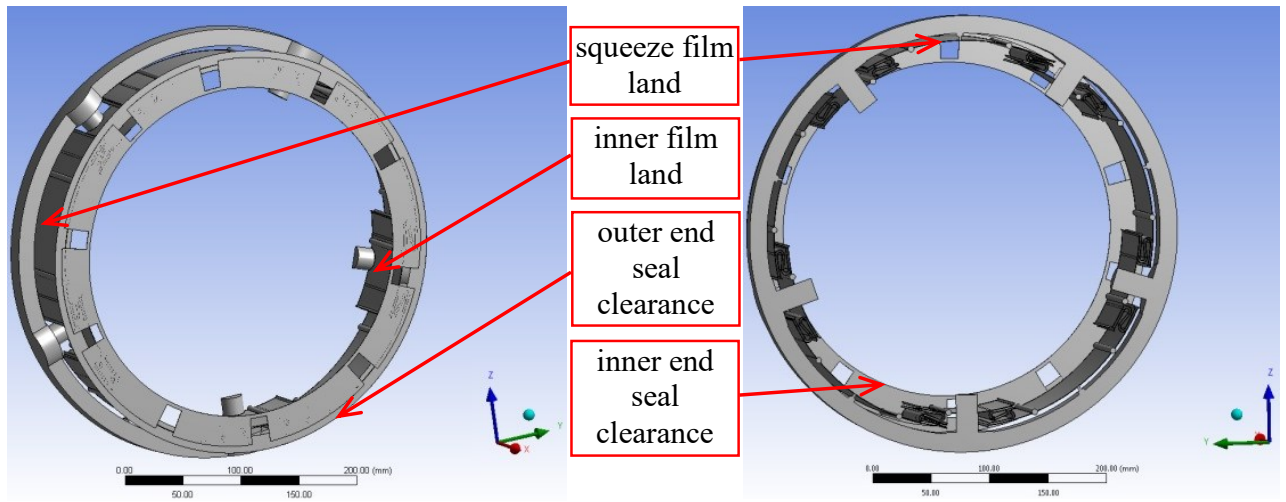

Fig. 4. Geometrical model of the fluid area in the segmented ISFD

A three dimensional CFD model is developed using the ANSYS CFX software. The geometry of the fluid area (Fig. 4) in the damper was prepared with the ANSYS Workbench software and the structured grid was generated with the Altair HyperMesh software.

Twenty divisions were used across the film thickness, 16 and 12 divisions were used across the outer and inner end seal clearance (Fig. 4), 360 and 230 divisions were used in the circumferential direction of the squeeze film land and of the inner damper land for one damper segment, and 120 divisions were used in the axial direction. A fine mesh (see Figs. 5, 6) was used to capture the high-velocity, respectively -pressure gradient. The total number of cells in the grid is approximately equal to 20 million. The grid portion of the segmented ISFD without the grid of the end seal clearance is presented in Fig. 5. The local grid of the end seal clearance is depicted in Fig. 6.

In this work, it is assumed that the orbit of the damper to be circular and whirls around the centre shifted to the outer damper ring. The displacement and velocity components for synchronous circular whirling of the damper orbit are defined by the following relations

$$
\begin{gathered}
y_{0}(t)=e \cos (\omega t), z_{0}(t)=e \sin (\omega t), \\
v_{0}(t)=-e \omega \sin (\omega t), w_{0}(t)=e \omega \cos (\omega t),
\end{gathered}
$$

where $y_{0}, z_{0}$ are the $y$ and $z$ components of the displacement, $v_{0}, w_{0}$ are the $y$ and $z$ components of the velocity, $\omega$ is the whirl frequency, and $e$ is the radius of the whirl orbit. Parameters of the synchronous circular whirling of the journal are defined as follows: the whirl frequency is $30 \mathrm{~Hz}(188.496 \mathrm{rad} / \mathrm{s})$ and the radius of whirl orbit is $2 \mu \mathrm{m}$.

The moving mesh method was not employed because the radius of whirl orbit is much smaller than the thickness $(0.4 \mathrm{~mm})$ of the squeeze film land. The fluid is assumed to be incompressible and Newtonian, the coefficient of viscosity is constant, there is no slip between the fluid and rings surface, and the gravity forces are not taken into account in the CFD model. The cavitation phenomenon is sometimes considered into account in the procedure for computation of the hydraulic force.

The fluid inertia and turbulence effects in SFDs are governed by the squeeze film Reynolds number [27, 28]

$$
R e=\frac{\rho \omega C^{2}}{\mu},
$$

where $\mathrm{C}$ is the thickness of the squeeze film land in the concentric position. 
The Reynolds number calculated according to equation (8) is equal to 0.84 and the Reynolds number corresponds to the onset of turbulence $\mathrm{Re} \approx 2000$ [27]. Therefore, its value is much smaller than the critical Reynolds number, and in ANSYS CFX the viscous model was set to laminar.

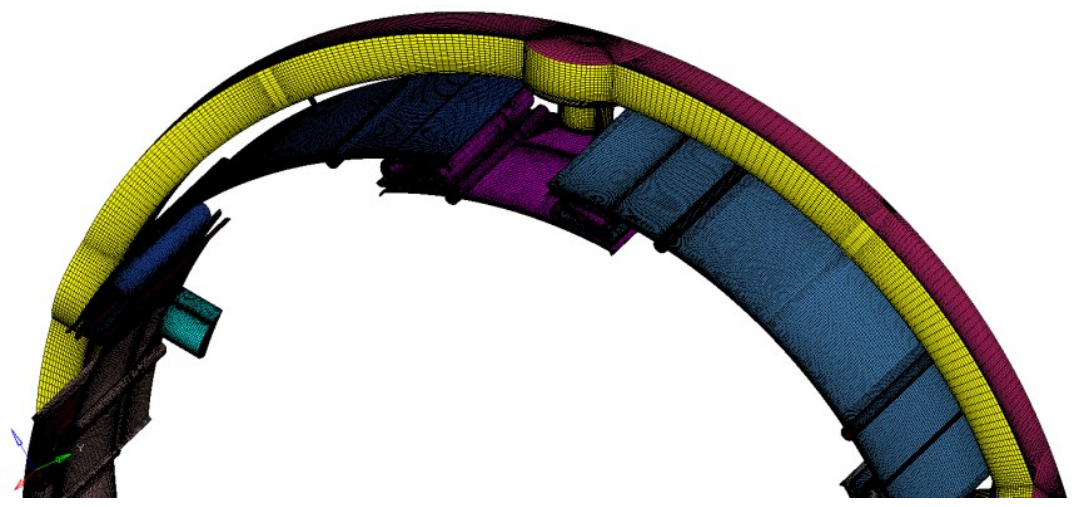

Fig. 5. The grid portion of the segmented ISFD (without the grid of the end seal clearance)

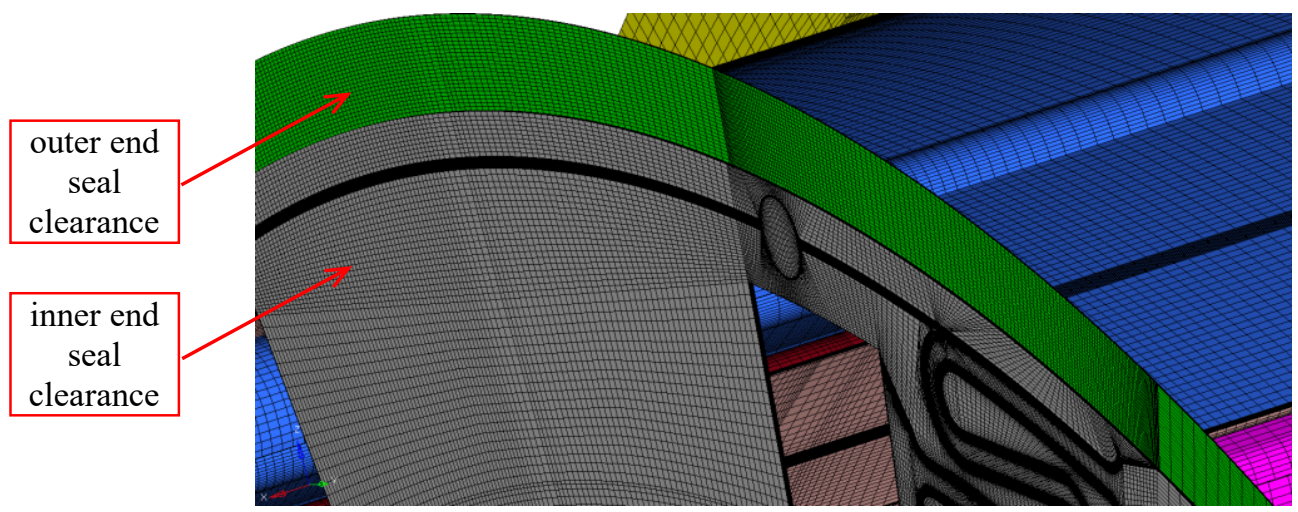

Fig. 6. The local grid of the end seal clearance

The pressure boundary conditions were defined in the form of the relative pressure (0 Pa, 0.05 MPa, 0.1 MPa, or $0.15 \mathrm{MPa}$, respectively) in the inlet (see Fig. 7a, ANSYS CFX, Boundary Type: Inlet), the relative pressure $(0 \mathrm{~Pa}, 0.05 \mathrm{MPa}, 0.1 \mathrm{MPa}$, or $0.15 \mathrm{MPa}$, respectively) at the outlet (see Fig. 7b, ANSYS CFX, Boundary Type: Opening), the relative atmospheric pressure $0 \mathrm{~Pa}$ at the outlet (see Fig. 7c, ANSYS CFX, Boundary Type: Opening), and the symmetry (see Fig. 7d) at the half of the damper length.
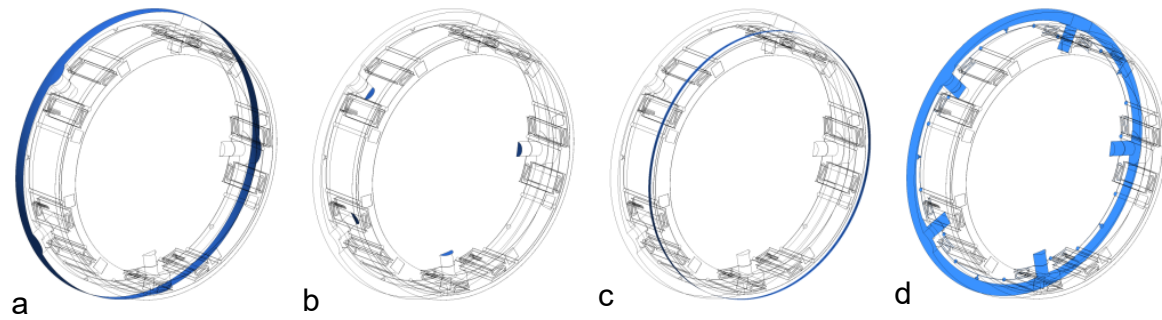

Fig. 7. The pressure and symmetry boundary conditions 
The velocity boundary conditions were defined in the form of the Cartesian velocity components $\left(u_{\mathrm{o}}=0 \mathrm{~m} \cdot \mathrm{s}^{-1}, v_{\mathrm{o}}, w_{\mathrm{o}}\right)$ on the inner surface of the squeeze film land, on the inner surface of the inner film land, and in the front surface of the end seal clearance (see Figs. 8a, 8b ANSYS CFX, Boundary Type: Inlet) and on the other surfaces were defined all Cartesian velocity components equal $0 \mathrm{~m} \cdot \mathrm{s}^{-1}$ (see Fig. 8c, ANSYS CFX, Boundary Type: Wall).
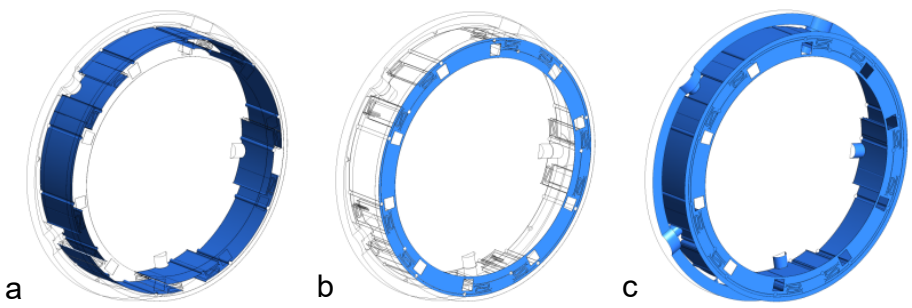

Fig. 8. The velocity boundary conditions

Navier-Stokes equations (1-3) and the continuity equation (4) with the corresponding boundary conditions are numerically solved with the use of the ANSYS CFX software.

The task was to determine the pressure and velocity distribution in the damper and the time dependency of the hydraulic force for the synchronous circular whirling of the journal.

\section{Results of the computational simulations}

The pressure and velocity distributions were computed at the specified moment of time equal to $t=0 \mathrm{~s}$, and the stationary analysis was used and repeated for several values of the supply pressures.

The pressure distribution in the segmented ISFD for four values of the supply pressure (0 Pa, 0.05 MPa, 0.1 MPa, and 0.15 MPa) is depicted in Fig. 9. It is clear that the total pressure is equal to the relative atmospheric pressure $0 \mathrm{~Pa}$ on the surface of the outlet boundary condition (Fig. 7c). However, in the case of the supply pressure equalling zero the damper pressure drops under the atmospheric one. The pressure distribution is almost constant through the thickness of the squeeze film land as well as the inner film land.
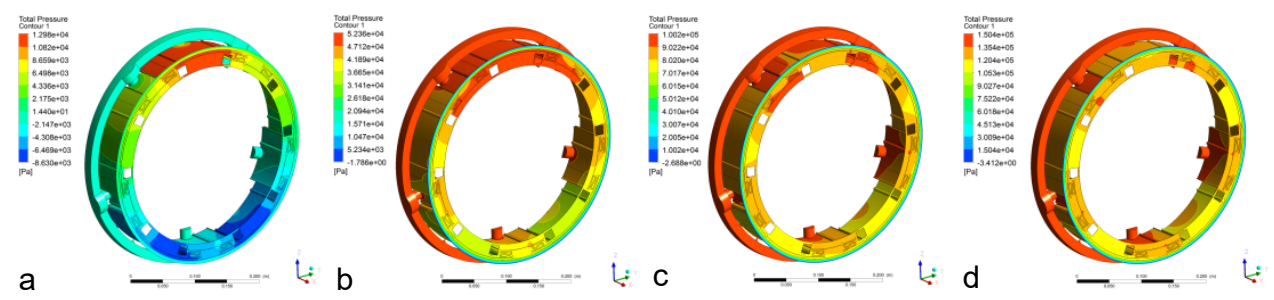

Fig. 9. Pressure distribution for $0 \mathrm{~Pa}$ (a), $0.05 \mathrm{MPa}$ (b), $0.1 \mathrm{MPa}$ (c), and $0.15 \mathrm{MPa}$ (d) of the supply pressure in the segmented ISFD

The velocity magnitude and the velocity vectors of the segmented ISFD for four values of the supply pressure $(0 \mathrm{~Pa}, 0.05 \mathrm{MPa}, 0.1 \mathrm{MPa}$, and $0.15 \mathrm{MPa})$ are drawn in Fig. 10 . The maximum value of the velocity magnitude grows with increasing the supply pressure and it is located in the oil supply nozzle of the squeeze film lands. If the supply pressure is nonzero, the oil outflow is around circumference of the damper outlet, see Fig. 10b-d. For zero supply pressure (Fig. 10a), the oil outflow is only one half of the circumference of the damper outlet. On the opposite circumferential outlet of the damper, the air is suctioned into the damper, see Fig. 9a, 10a. 

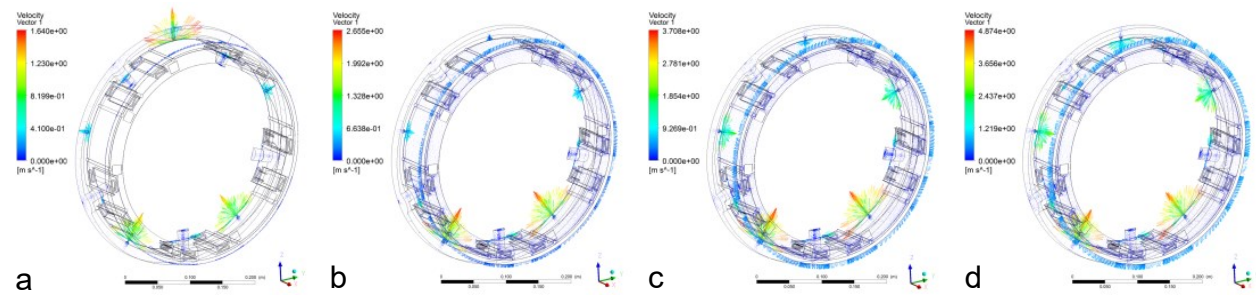

Fig. 10. The velocity magnitude and the velocity vectors for $0 \mathrm{~Pa}$ (a), $0.05 \mathrm{MPa}(\mathrm{b}), 0.1 \mathrm{MPa}$ (c), and $0.15 \mathrm{MPa}(\mathrm{d})$ of the supply pressure in the segmented ISFD

For the computation of the time dependence of the hydraulic force, the transient analysis is employed. The time history of the components of the hydraulic force in the horizontal (y) and vertical (z) directions during 2 periods is depicted in Fig. 11. It is evident that the components of the hydraulic force (Fig. 11a) have the mean value of almost zero and that the total hydraulic force is slightly varying in time (from $773 \mathrm{~N}$ to $788 \mathrm{~N}$ ).
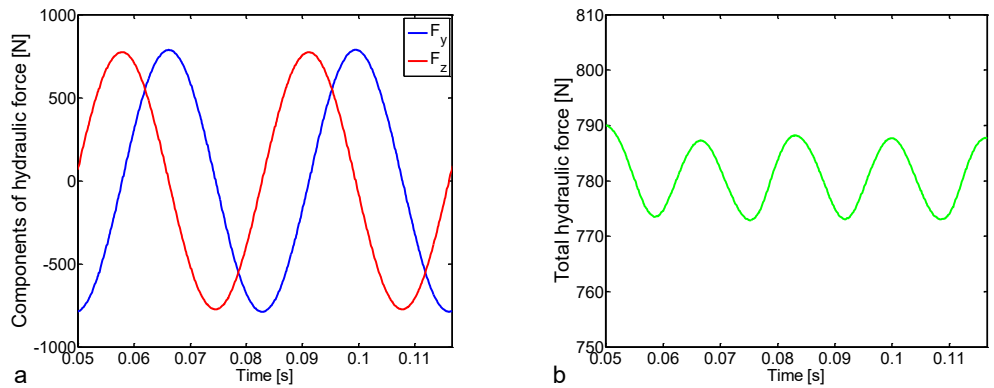

Fig. 11. Time history of the components of the hydraulic force (a) and total hydraulic force (b) in the segmented ISFD without the supply pressure

According to the assumption of the non-cavitated and cavitated operational regimes of the ISFD damper, the hydraulic forces are compared in Fig. 12. In the cavitated region, it is assumed that the pressure distribution is equal to the pressure in the ambient space and the hydraulic forces are computed with the use of the equation (5). It is obvious that the cavitation has a strong effect on the horizontal (y) component of the hydraulic force (Fig. 12a) and the total hydraulic force (Fig. 12b). In the cavitated regime, the mean value of the horizontal hydraulic force has non-zero mean value and the total hydraulic force is significantly varying in time.
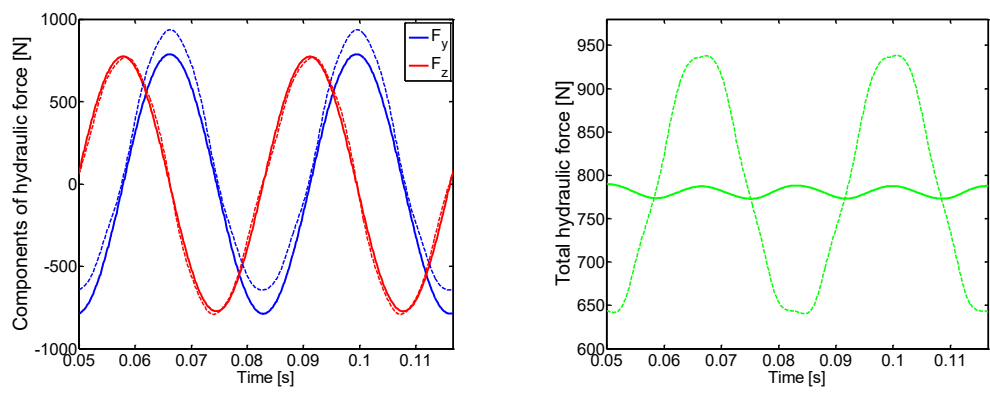

Fig. 12. Time history of the components of the hydraulic force (a) and total hydraulic force (b) in segmented ISFD without the supply pressure (solid line - cavitation not considered, dashed line cavitation considered) 
The time history of the components of the hydraulic force and the total hydraulic force during 3 periods is shown in Fig. 13. Due to the non-zero supply pressure and the eccentric position of the damper rings, the mean value of the vertical $(z)$ component of the hydraulic force is non-zero (-390 N, see Fig. 13a). On the contrary, in the case of zero supply pressure of the damper, the total hydraulic force is significantly variable in time (from $433 \mathrm{~N}$ to $1224 \mathrm{~N}$ ). The mean value of the total hydraulic force for the supply pressure $0 \mathrm{~Pa}$ is equal to $780.5 \mathrm{~N}$ (Fig. 11b) and for one $0.15 \mathrm{MPa}$ is equal to $828.5 \mathrm{~N}$ (Fig. 13b).
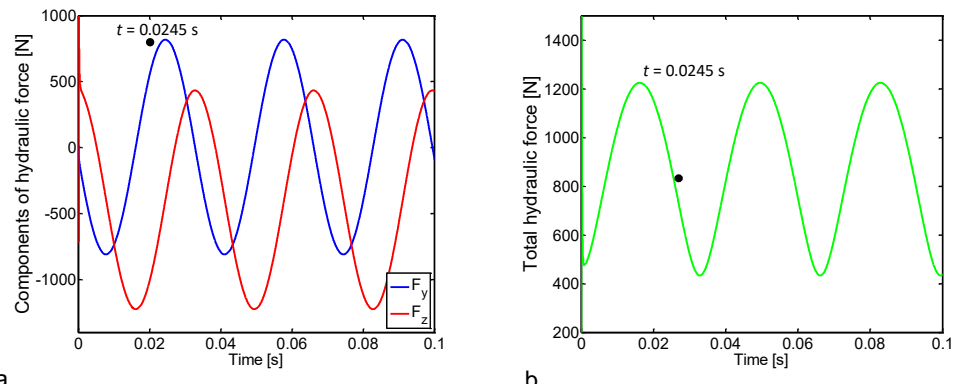

Fig. 13. Time history of the components of the hydraulic force (a) and total hydraulic force (b) in segmented ISFD with the supply pressure $0.15 \mathrm{MPa}$

The pressure distribution (Fig. 14a) and the velocity magnitude and velocity vectors (Fig. 14b) at a time of $0.0245 \mathrm{~s}$ that corresponds to the maximum value of the horizontal (y) hydraulic force component are depicted in Fig. 14. It is evident that the pressure distribution (Fig. 14a) is almost positive in the entire damper. The maximum value of the velocity magnitude (Fig. 14b) is similar to the stationary one, see Fig. 10d.
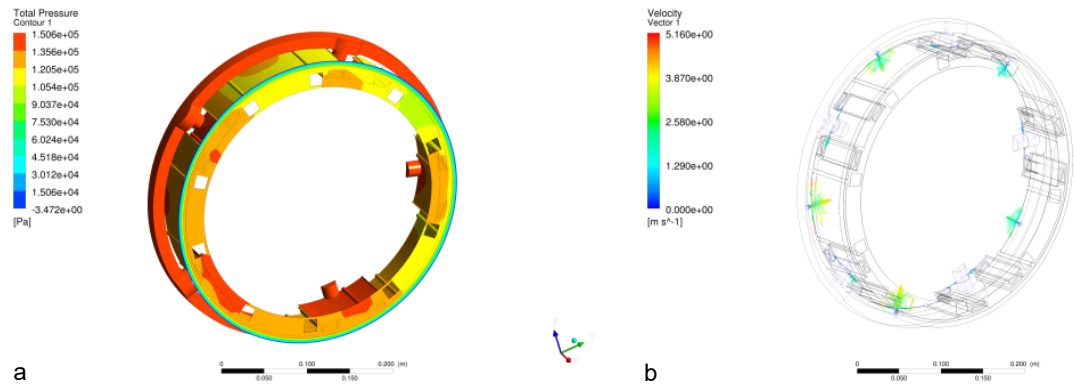

Fig. 14. The pressure distribution (a) and the velocity magnitude (b) at time $0.0245 \mathrm{~s}$ in segmented ISFD with the supply pressure of $0.15 \mathrm{MPa}$.

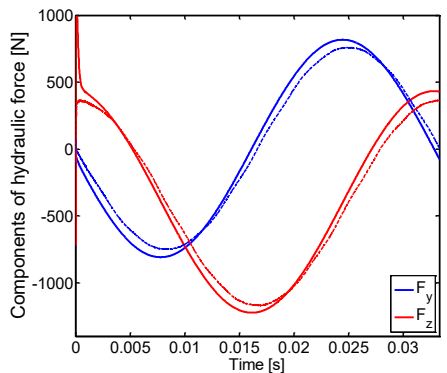

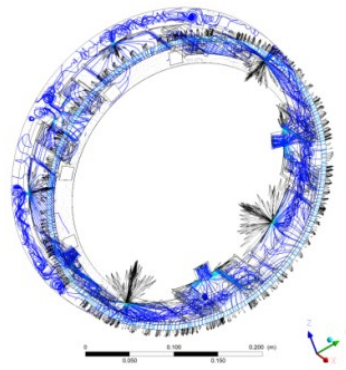

$\mathrm{b}$

Fig. 15. Time history of the components of the hydraulic force (solid line $\rho=870 \mathrm{~kg} \cdot \mathrm{m}^{-3}$, dashed line $\rho=1 \mathrm{~kg} \cdot \mathrm{m}^{-3}$ ) (a) and the streamline flow (b) in the segmented ISFD with the supply pressure of $0.15 \mathrm{MPa}$. 
The effect of the inertia of the oil was studied on the components of the hydraulic force, which are shown in Fig. 15a. The smaller inertia effect was reached by the reduction of the oil density (for the oil density smaller than $1 \mathrm{~kg} \cdot \mathrm{m}^{-3}$, the convergence has not been satisfactory). As a result of the inertia, the hydraulic forces are increased by $8 \%$, and the time dependence of the components of the hydraulic force has the phase shift.

The streamlines flow for forward and backward directions at a time of $0.0333 \mathrm{~s}$, and the velocity vectors are depicted in Fig. 15b. The dominant flow is located in the oil supply nozzle of the squeeze film lands, and the oil outflow is in the outlet (which is $0.1 \mathrm{~mm}$ thin film of the oil).

\section{Conclusions}

The CFD model of the segmented ISFD has been developed. The simplified CFD model of the damper was validated by comparing the results with the theoretical solution of the short approximation of SFD. The pressure and velocity distributions and the hydraulic forces in the segmented ISFD were computed. The effect of the supply pressure on the performance of the damper was studied by means of the stationary analysis. It was found that the supply pressure and the inertia of the oil have the influence on the pressure distribution and the hydraulic forces in the segmented ISFD.

Currently, the authors are working on the development of the computation procedure for the identification of dynamic mass, damping, and stiffness coefficients of the segmented ISFD.

This work has been supported by The Ministry of Education, Youth and Sports from the National Programme of Sustainability (NPS II) project „IT4Innovations excellence in science - LQ1602“, by the research organization conceptual development project (project no. RVO: 61388998), and by the European Union's Horizon 2020 research and innovation programme under grant agreement No. 653941. The support is highly acknowledged.

\section{References}

1. J. Tůma, J. Škutová, Proceedings of the 13th International Carpathian Control Conference, (High Tatras, Slovakia, 2012)

2. J. Tůma, J. Šimek, J. Škuta, J. Los, Mech. Syst. Signal Process. 36, 2 (2013)

3. A. Tonoli, N. Amati, M. Silvagni, Electromechanical dampers for vibration control of structures and rotors, Vibration control, (InTech, Croatia, 2010)

4. A. Tonoli, N. Amati, A. Bonfitto, M. Silvagni, B. Staples, E. Karpenko, J. Eng. Gas Turbines Power 132, 11 (2010)

5. Y. Kligerman, O. Gottlieb, J. Vibr. Acoust. 120, 4 (1998)

6. A. El-Shafei, Dynamics of rotors incorporating squeeze film dampers, (Massachusetts Institute of Technology, USA, 1988)

7. J.M. Vance, Rotordynamics of turbomachinery, (John Willey \& Sons, USA, 1988)

8. L.D. Pietra, G. Adiletta, Shock Vib. Dig. 34, 1 (2002)

9. L.D. Pietra, G. Adiletta, Shock Vib. Dig. 34, 2 (2002)

10. A. El-Shafei, J. Eng. Gas Turbines Power 124, 3 (2002)

11. H.R. Heidari, P. Safarpour, Mech. Mach. Theory 105, November (2016)

12. A. Bouzidane, M. Thomas, Comput. Struct. 86, 3-5 (2008)

13. Y. Sun, M. Thomas, J. Vib. Control 17, 8 (2011) 
14. C. Carmignani, P. Forte, E. Rustighi, Smart Mater. Struct. 15, 1 (2006)

15. G.-J. Wang, N. Feng, G. Meng, E.-J. Hahn, J. Intell. Mater. Syst. 17, 4 (2006)

16. J. Zapoměl, P. Ferfecki, P. Forte, Smart. Mater. Struct. 21, 10 (2012)

17. P. Ferfecki, J. Zapoměl, J. Kozánek, Adv. Eng. Softw. 104, February (2017)

18. J. Zapoměl, P. Ferfecki, P. Forte, Appl. Math. Model. 52, December (2017)

19. J. Zapoměl, P. Ferfecki, J. Vib. Acoust. 140, 1 (2018)

20. F. Zeidan, L. San Andrés, J.M. Vance, Proceedings of the Twenty-Fifth Turbomachinery Symposium, (Texas A\&M University, College Station, TX, USA, 1996)

21. O. de Santiago, L. San Andrés, J. Oliveras, J. Eng. Gas Turbines Power 121, 4 (1999)

22. O. de Santiago, L. San Andrés, J. Eng. Gas Turb. Power 125, 4 (2003)

23. B. Ertas, V. Cerny, J. Kim, V. Polreich, J. Eng. Gas Turb. Power 137, 5 (2015)

24. B. Ertas, A. Delgado, J. Moore, Proceedings of ASME Turbo Expo 2017: Turbomachinery Technical Conference and Exposition, (Charlotte, NC, USA, 2017)

25. A.Z. Szeri, Tribology: Friction, lubrication, and wear, (Hemisphere Publishing Corporation, USA, 1980)

26. F.Y. Zeidan, J.M. Vance, J. Tribol.-T. 32, 1 (1989)

27. J.A. Tichy, Proceeding of ASME 1983 International Gas Turbine Conference and Exhibit, (Phoenix, Arizona, USA, 1983)

28. L. San Andrés, J.M. Vance, NASA. Lewis Research Center Rotordynamic Instability Problems in High-Performance Turbomachinery, (Texas A\&M, USA, 1984) 